\title{
Unobtrusive Online Monitoring of Sleep at Home
}

\author{
Joonas Paalasmaa, Mikko Waris, Hannu Toivonen, Lasse Leppäkorpi, and Markku Partinen
}

\begin{abstract}
We describe an online sleep monitoring service, based on unobtrusive ballistocardiography (BCG) measurement in an ordinary bed. The novelty of the system is that the sleep tracking web application is based on measurements from a fully unobtrusive sensor. The BCG signal is measured with a piezoelectric film sensor under the mattress topper, and sent to the web server for analysis. Heart rate and respiratory variation, activity, sleep stages, and stress reactions are inferred based on the signal. The sleep information is presented to the user along with measurements of the sleeping environment (temperature, noise, luminosity) and user-logged tags (e.g. stress, alcohol, exercise). The approach is designed for longterm use at home, allowing users to follow the development of their sleep over months and years. The service has also a medical use, as sleep disorder patients can be measured for long periods before and after interventions.
\end{abstract}

\section{INTRODUCTION}

$\mathbf{M}$ EASUREMENT and tracking of sleep length and sleep quality, as well as measuring different events (apneas, periodic limb movements, movement arousals) during sleep over long time periods can help improve one's sleep and well-being. Such methods could also be used in followup of patients with various sleep disorders. In this paper, we present a novel web application for monitoring the length and quality of sleep. The measurement is based on an unobtrusive sensor that does not deteriorate sleep quality.

As an overview, the service works as follows. A thin piezoelectric force sensor, placed under the mattress topper, measures the mechanical vibrations of the person. These signals are automatically sent to a web server for analysis. The server first infers the heart rate, respiration rate, and activity information from the signals (as the data is received). Based on these results, the server then analyzes the sleep (as batch processing, typically in the morning). Finally, various aspects of sleep are presented to the user on a web site. A daily view shows features such as sleep stages, whereas a timeline view shows trends and long-term changes in sleep quality.

Inferring sleep stages based on the measurement of heart rate variability, respiration rate variability and/or activity

Manuscript received March 28, 2012.

J. Paalasmaa is with Beddit.com Ltd, Espoo, Finland, with Department of Computer Science, University of Helsinki, Helsinki, Finland, and with Department of Signal Processing, Tampere University of Technology, Tampere, Finland (email: joonas.paalasmaa@beddit.com).

M. Waris and L. Leppäkorpi are with Beddit.com Ltd, Espoo, Finland.

H. Toivonen is with Department of Computer Science, University of Helsinki, Helsinki, Finland.

M. Partinen is with Helsinki Sleep Clinic, Vitalmed Research Center, Helsinki, Finland and with Department of Clinical Neurosciences, Helsinki University Central Hospital, Helsinki, Finland. has been proposed by many research groups [1]-[5]. These approaches are based on extracting various features from these parameters and using a classifier to map them to sleep stages.

Measurement of heart rate variability using sensors in the bed has a long history. The method, known as ballistocardiography (BCG), attracted much interest from late 1930s to late 1950s in the cardiovascular diagnosis community. Those efforts faded away when new tools such as electrocardiography were developed [6]. The interest in BCG has increased again recently, because it enables long-term measurement of heart rate variability without electrodes, or wearable sensors, or any other discomfort.

The precision of mechanical bed sensors for the analysis of heart rate variability [7] and respiratory variation [8] has already been shown to be good. Various sensors have been proposed, for example, a pillow sensor [9], a bed post sensor [8], [10], as well as different kinds of mattress sensors [11], [12]. Using an unobtrusive measurement method and sending the results over the Internet in real time has been proposed for the measurement of heart rate and respiratory variation [13] as well as for actigraphy based sleep/wake classification [14].

There are several alternative methods for measuring sleep quality and diagnosing sleep disorders. We briefly review them next.

Polysomnography (PSG) is the standard method for scoring of sleep and involves the measurement of electroencephalography, submental electromyography, and electrooculography, at a minimum. Additional sensors are needed in diagnostic sleep recordings. These include measurement of airflow, breathing movements, oxygen saturation, limb movements, and electrocardiography. PSG can be considered as the golden standard in sleep medicine. It provides precise information about sleep but is quite expensive and not commonly available.

Another popular diagnosis method is wrist actigraphy. There, the movement activity of the patient is monitored typically during one week, 24 hours a day. The sampling rate varies between 32 and $256 \mathrm{~Hz}$ depending of the manufacturer and aim of the measurement. The advantages of actigraphy over PSG are that the patient can sleep undisturbed and that the measurement period is longer. However, as actigraphy only measures movement, it has limited sleep diagnostic capability. According to the American Academy of Sleep Medicine (AASM), actigraphy is a valid and standard way to assist in determining sleep patterns in normal, healthy adult populations, and it can be used also in patients suspected of certain sleep disorders, such as circadian sleep-wake 
disorders. [15]

Recently, new devices for measurement of sleep quality have emerged. These devices are usually based on actigraphy, they are not intended for diagnosis, and they have no medical approval. Instead, they are inexpensive and are sold directly to customers for self-help use. Zeo Sleep Manager measures both movement and EEG, and its accuracy has been validated using PSG [16]. Fitbit, BodyMedia FIT, Jawbone UP, SleepTracker, LARK, and WakeMate are based on wrist actigraph measurement. Many of these devices have a web application for viewing the measurements and a smartphone interface.

There also are some telemedicine applications for sleep monitoring [17], [18]. They are typically based on PSG, which is measured at home using EEG, EMG etc. The signals are sent to a sleep doctor for interpretation.

The contribution of this paper is a fully automated web application for sleep analysis at home. The novelty of the approach is that sleep is measured with an unobtrusive method and that sleep measurements are presented to the user in an intuitive way. We next give an overview of the methods used. We then describe the self-help sleep analysis application, as well as a medical application based on longterm monitoring of sleep.

\section{METHODS}

\section{A. Measurement}

A flexible piezo-electric force sensor is used for measuring the mechanical activity of the sleeping person. The sensor measures 4 by $70 \mathrm{~cm}$ and is $0.4 \mathrm{~mm}$ thick (Fig. 1). It is preferably placed between the mattress topper and mattress, or under the mattress if no topper is used. A signal excerpt from the sensor is shown in Fig. 2.

The sensor is attached to a custom-made miniature Linux computer that carries out the analog-to-digital conversion and sends the data to the Internet over a wired or wireless connection.

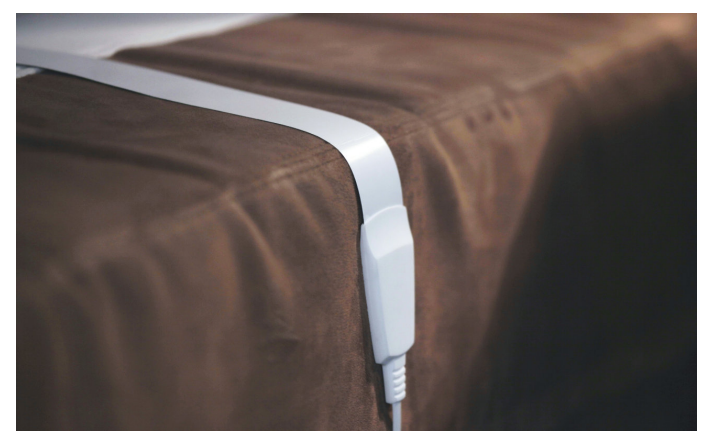

Fig. 1. The sensor.

The force signal from the sensor is sampled at $140 \mathrm{~Hz}$. Additionally, temperature, ambient noise level, and brightness are measured periodically. They are used to evaluate the quality of the sleeping environment. This information, about 300 kilobytes of compressed data per hour, is sent to the server as it is measured.

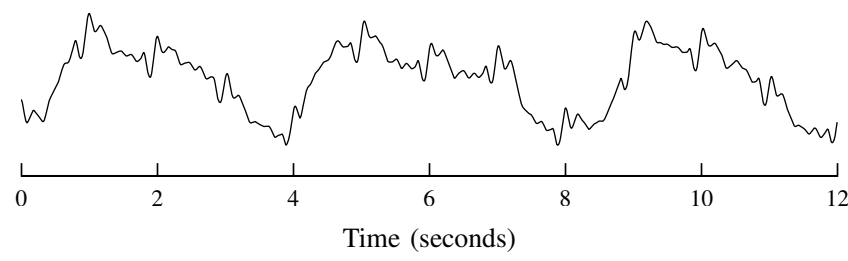

Fig. 2. A 12-second signal excerpt. The low-frequency phenomenon with around four-second period is respiration. The heartbeat is the fluctuation that recurs around every second.

A flowchart of the analyses that follow is shown in Fig. 3 . We give an overview of these steps next.

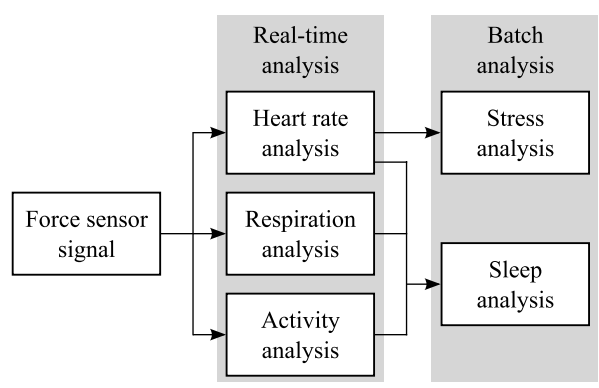

Fig. 3. Analysis flowchart.

\section{B. Signal analysis}

The heart rate variability is analyzed with a novel algorithm. We outline it here, but full details are outside the scope of this paper. The algorithm first forms a heartbeat shape template using complete-link clustering [19]. Heartbeat intervals are then detected with a generative approach, where the signal is modeled as a summation of transformed heartbeat vectors [19]-[21]. In this model, the $j$ th sample in the signal is expressed as

$$
s_{j}=\left(\sum_{i=1}^{N} \mathbf{x}_{i}\left[j-t_{i}\right]\right)+\epsilon
$$

where $\mathbf{x}_{i}$ is the $i$ th heartbeat vector, $t_{i}$ is the position of that heartbeat, $\mathbf{x}_{i}[k]$ denotes the $k$ th item of vector $\mathbf{x}_{i}$, and $\epsilon$ is noise. Further, each $\mathbf{x}_{i}$ is modeled using the linear latent variable model

$$
\mathbf{x}_{i}=\mathbf{w} z_{i}+\boldsymbol{\mu}+\epsilon
$$

where $\boldsymbol{\mu}$ represents the mean heartbeat shape, $\mathbf{w}$ is the direction of variation (a vector), and $z_{i}$ is the magnitude of variation (a scalar) [20]. The variation of the heartbeat shape is caused mainly by respiration. The generative approach for heartbeat detection selects those heartbeat positions that minimize the residual error $\epsilon$.

The algorithm has been validated with a 40-patient group at Helsinki Sleep Clinic, Helsinki, Finland ${ }^{1}$. Preliminary results from the validation show over $98 \%$ precision with all the patients even though some of them had very challenging

\footnotetext{
${ }^{1}$ The study was approved by the Coordinating Ethics Committee of the District Hospital of Helsinki and Uusimaa.
} 
signals due to pathologies. However, for this reason, there were larger differences in recall. The lowest recall, 20\%, was seen in a very arrhythmic patient. 32 patients had over $50 \%$ recall and 16 over $80 \%$ recall. An exemplary heart rate variation graph along with the reference is shown in Fig. 4. Full validation will be published elsewhere.

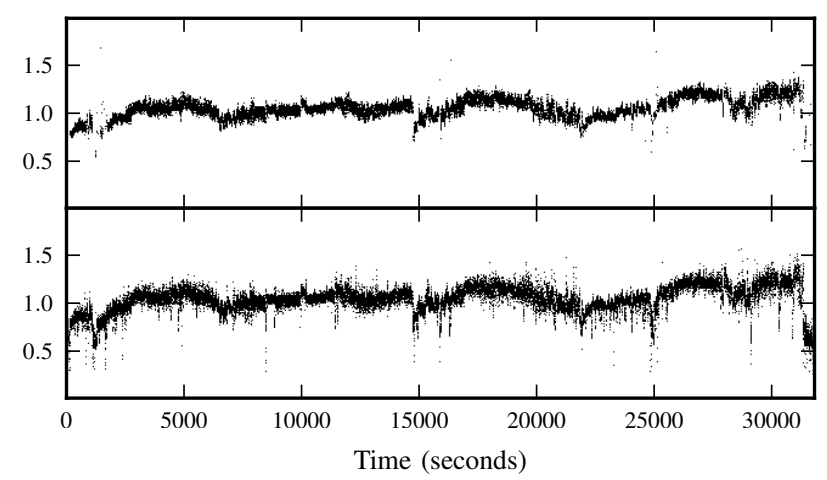

Fig. 4. Heartbeat intervals (in seconds) detected from BCG (top plot) are compared with an ECG reference (bottom plot). In this case, precision is $99.96 \%$ and recall $88.73 \%$.

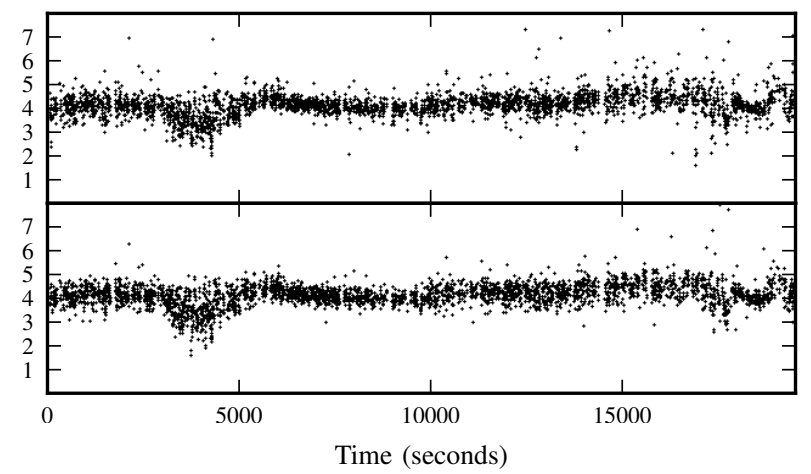

Fig. 5. Respiration cycle lengths (in seconds) detected from BCG (top plot) are compared with an airflow reference (bottom plot) (from [8]).

The method presented in [8] is used for the respiration rate variability analysis. The length of every respiration cycle is detected, which makes it possible to quantify respiratory variation precisely. We omit the details. Fig. 5 shows a comparison with a reference.

The activity information is analyzed so that discrete events of activity are detected from the force signal. The result is called the binary actigram.

\section{Sleep analysis}

When the server receives the signal from the sensor, heart rate variability, respiration rate variability, and binary actigram are analyzed immediately. Based on those results, the following sleep analyses are calculated in the morning:

- Sleep staging into the following phases: wake, REM sleep, light sleep and deep sleep

- Stress reactions (based on heart rate variability) [22]

- Heart rate curve and average heart rate

- Restlessness index
The sleep staging is carried out by an algorithm that utilizes heart rate variation, respiratory variation and activity information (cf. [1]-[5]). The validation of the algorithm is under progress.

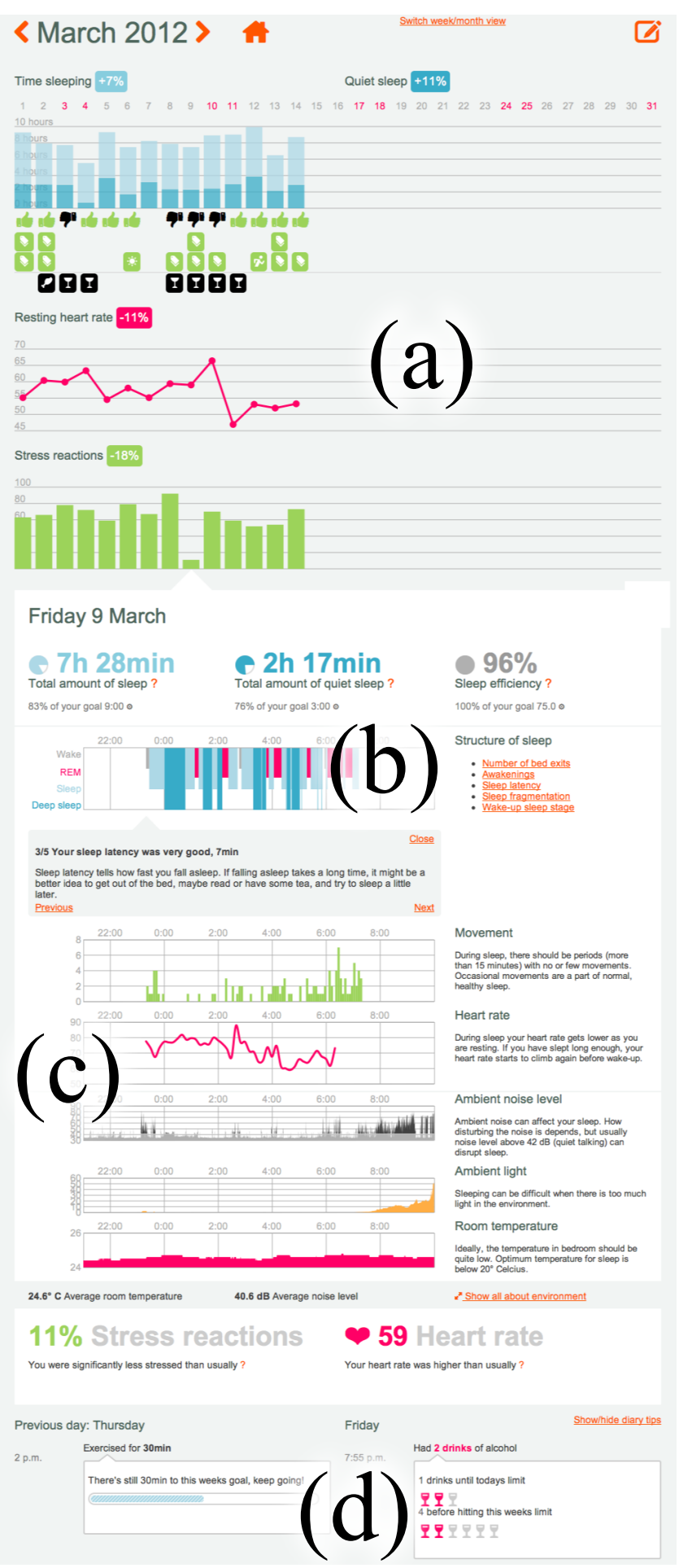

Fig. 6. (a) The timeline and navigation view containing sleep times, user entered tags, resting heart rates, and relative stress reaction values for each day. (b) Night summary figures. (c) Detailed night information, including sleep stages and a guide to their interpretation, actigraphy, heart rate, ambient noise, ambient luminosity, room temperature, relative stress reactions and resting heart rate figures. (d) Logged entries for the previous and following day along with their comparison to the intended goal. 


\section{SELF-HELP USE CASE: SLEEP DISCOVERY WEB APPLICATION}

\section{A. Purpose and usage}

The purpose of the web application is to let users make discoveries about their sleep and lifestyle. Sleep information is presented so that the user can easily notice the relevant features of her sleep.

The sleep of a single night is presented with detailed structure (lower half of Fig. 6). The main feature is the hypnogram, where sleep is divided into wakefulness, REM sleep, light sleep and deep sleep. From the hypnogram, simple features that describe the night succinctly are calculated: total amount of sleep, sleep efficiency, number of bed exits and number of awakenings.

The overall restlessness of sleep is visualized with a plot that shows how much movement there is in different parts of the night. Restlessness is calculated by summing actigram events over 5-minute time intervals.

The heart rate information is shown as a trend curve and as a single resting heart rate number that corresponds to the lowest point on the curve. The curve makes it possible to discover interesting features in heart rate. For example, if the user consumes alcohol in the evening, a high heart rate will be detected at the beginning of the night. The resting heart rate is a good number for display in the timeline view, as it summarizes the whole night.

Stress reactions are estimated from heart rate variability [22]. Estimating absolute stress is difficult due to interpersonal physiological variability, so stress is displayed as a relative number between $0 \%$ and $100 \%$. There, $50 \%$ corresponds to the average stress reaction level and differences from the average decrease or increase the percentage from 50 .

Temperature, brightness and noise level measurements are shown as plots for the period of the whole night. The user can see sleeping environment problems, such as too high a noise level, from the measurements.

The other way to visualize sleep is a timeline view, where sleep information from a time period is summarized. In addition to displaying sleep amounts for each night in the period, summary figures and relevant cyclic phenomena are shown. For a period of one week, the circadian rhythm is shown, and for a period of a month, the weekly sleep rhythm (week versus weekend). The monthly timeline view is shown in the upper half or Fig. 6.

\section{B. Engaging users to learn}

No self-help program can help if it is left unused. To make use as easy as possible, the measurement device is designed to work fully automatically and the user is reminded to check her sleep with daily emails.

Each morning, the user receives an email with last night's sleep information in a concise form. An easy-to-use mechanism is provided with the email for logging daytime feeling: by clicking a link the user can tell whether she feels refreshed or tired. After that, the user is also asked to describe the previous evening with simple tags. The tags represent things that may have affected sleep, such as stress, alcohol, caffeine, television and exercise.

The logged information is shown in the timeline view along with sleep information (Fig. 6, (a)). This helps to recognize, for example, that stress in the evening leads to tired daytime feeling.

\section{Motivating with daily and weekly goal setting}

Setting goals is a key feature of the web application. Goals can be set for sleep time and for things that the user has logged. The rationale for self-specified goals is twofold. First, it is difficult to come up with a sensible sleep time recommendation for the user, because sleep need is highly individual. We assume that the user can set sensible sleep time goals after some use of the system.

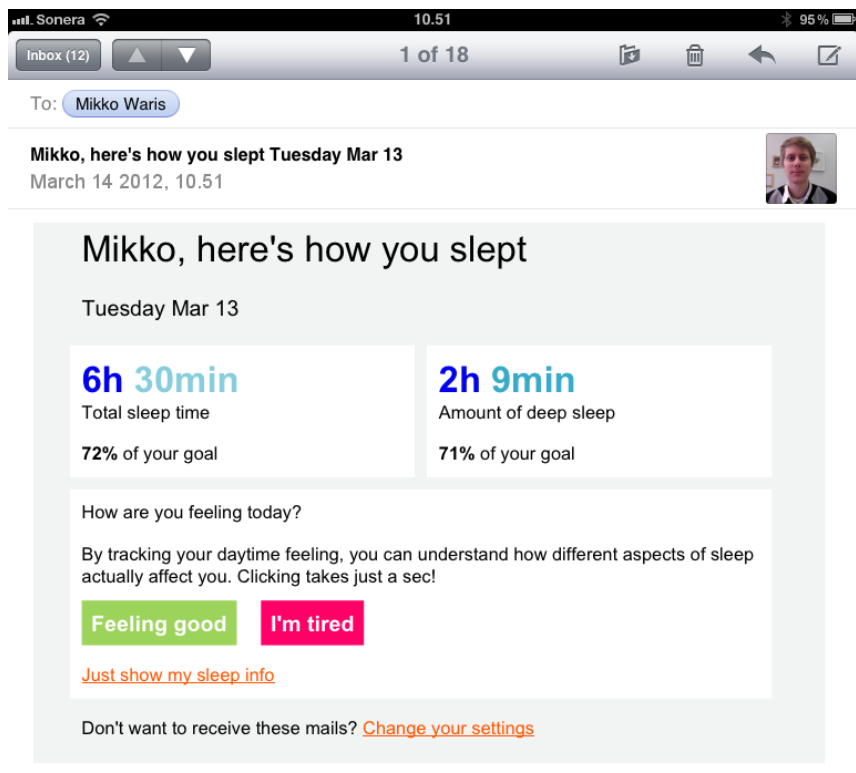

Fig. 7. Logging daytime feeling.

Secondly, the goal setting provides motivation for the user to improve lifestyle. By setting goals or limits for exercise or alcohol usage, the user creates a goal intention. While people often have vague intentions such as "I will quit smoking one day" or "I will lose weight this year", the web application helps the user enter more concrete goals and translates them into intentions: "This week, I will exercise at least 2 hours" or "I will not have more than one alcohol drink per day". It has been shown that establishing intentions significantly improves goal achievement rates [23].

The goals and performance in achieving them are visualized to user as shown in Fig. 6, (d).

Goal setting could further be improved by structuring the goal intentions so that they would include a plan about how the user intends to implement the goals and providing reward in form of an achievement badge or sharing the successfully achieved goals in social media. 


\section{MEDiCAL APPLICATION: LONG-TERM MONITORING}

The presented sleep measurement method makes it possible to monitor patients with sleep problems for months or even years in their normal sleeping environment.

The presentation of sleep information in medical use needs to be different from the self-help application. In medical use, the users of the web application are sleep doctors who interpret measurements from their patients. The following information is visualized: time in bed, actigram, heart rate and respiration rate, as well as measurements of the sleeping environment. Sleep stages are not shown, because sleep doctors are used to EEG-based sleep stages rather than the wake, REM, light sleep, deep sleep -classification of cardio-respiratory sleep staging. For analysis of sleep-related breathing disorders (SRBD), the raw force sensor signal is provided to the sleep doctor. The measured signal is similar to a signal from a static charge sensitive bed, which have been used for preliminary apnea analysis [24].

The advantages of the presented approach over actigraphy in medical use are: 1) measurement is unobtrusive, 2) sleep staging is more precise, and 3) respiratory and cardiac information is measured in addition to activity. The drawback compared to actigraphy is that daytime activity cannot be measured.

We believe that the method is particularly suitable for diagnosing periodic hypersomnias, where long measurement terms are required for characterizing the circadian patterns.

\section{CONCLUSions}

A web application for monitoring sleep at home with an unobtrusive sensor is presented. The self-help use case makes it possible to track the quality and quantity of sleep as well as how lifestyle affects sleep, with little effort. The user of the application can, for example, notice that alcohol consumption in the evening leads to being tired the next day. That hopefully results in positive lifestyle changes.

Medical use of the unobtrusive sleep measurement approach enables new ways to diagnose sleep disorders. The care of a sleep disorder patient can be started by measuring sleep with the unobtrusive sensor, instead of wrist actigraphy, for a few weeks, after which the sleep doctor interprets the measurements and does a more detailed diagnosis using polysomnography, if that is required. The measurement continues over the whole period of care and follows up the effectiveness of treatments to the diagnosed sleep disorders.

\section{REFERENCES}

[1] S. Redmond, P. de Chazal, C. O’Brien, S. Ryan, W. McNicholas, and C. Heneghan, "Sleep staging using cardiorespiratory signals," Somnologie, vol. 11, no. 4, pp. 245-256, 2007.

[2] W. Karlen, C. Mattiussi, and D. Floreano, "Improving actigraph sleep/wake classification with cardio-respiratory signals," in 30th Annual International Conference of the IEEE Engineering in Medicine and Biology Society, 2008, pp. 5262-5265.

[3] D. Mack, J. Patrie, R. Felder, P. Suratt, and M. Alwan, "Sleep assessment using a passive ballistocardiography-based system: Preliminary validation," in 31st Annual International Conference of the IEEE Engineering in Medicine and Biology Society, 2009, pp. 4319-4322.
[4] G. Chung, B. Choi, J. Lee, J. Lee, D. Jeong, and K. S. Park, "REM sleep estimation only using respiratory dynamics," Physiological Measurement, vol. 30, no. 12, pp. 1327-1340, 2009.

[5] B. H. Choi, G. S. Chung, J. Lee, D. Jeong, and K. S. Park, "Slowwave sleep estimation on a load-cell-installed bed: a non-constrained method," Physiological Measurement, vol. 30, no. 11, pp. 1163-1170, 2009.

[6] N. T. Smith, "Ballistocardiography," in Noninvasive cardiology; clinical cardiology monographs, A. M. Weissler, Ed. New York: Grune \& Stratton Inc., 1974.

[7] C. Bruser, K. Stadlthanner, S. de Waele, and S. Leonhardt, "Adaptive Beat-to-Beat heart rate estimation in ballistocardiograms," IEEE Transactions on Information Technology in Biomedicine, vol. 15, no. 5 , pp. 778-786, 2011.

[8] J. Paalasmaa, L. Leppäkorpi, and M. Partinen, "Quantifying respiratory variation with force sensor measurements," in 33rd Annual International Conference of the IEEE Engineering in Medicine and Biology Society, 2011, pp. 3812-3815.

[9] J. Cha, H. Choi, J. Shin, and K. Lee, "Unconstrained respiration and heart rate monitoring system based on a PPG pillow during sleep," in 30th Annual International Conference of the IEEE Engineering in Medicine and Biology Society, 2008, pp. 3224-3226.

[10] M. Brink, C. H. Müller, and C. Schierz, "Contact-free measurement of heart rate, respiration rate, and body movements during sleep,' Behavior Research Methods, vol. 38, no. 3, pp. 511-521, 2006.

[11] J. L. Jacobs, P. Embree, M. Glei, S. Christensen, and P. K. Sullivan, "Characterization of a novel heart and respiratory rate sensor," in 26th Annual International Conference of the IEEE Engineering in Medicine and Biology Society, vol. 1, 2004, pp. 2223-2226.

[12] Y. Chee, J. Han, J. Youn, and K. Park, "Air mattress sensor system with balancing tube for unconstrained measurement of respiration and heart beat movements," Physiological Measurement, vol. 26, no. 4, pp. 413-422, 2005.

[13] X. Zhu, W. Chen, Z. Tang, T. Nemoto, and D. Wei, "Automatic home care system for monitoring HR/RR during sleep," in 30th Annual International Conference of the IEEE Engineering in Medicine and Biology Society, 2008, pp. 522-525.

[14] C. Cheng, Y. Hsu, and C. Young, "Development of a portable device for telemonitoring of physical activities during sleep," Telemedicine and e-Health, vol. 14, no. 10, pp. 1044-1056, 2008.

[15] T. Morgenthaler, C. Alessi, L. Friedman, J. Owens, V. Kapur, B. Boehlecke, T. Brown, A. Chesson Jr., J. Coleman, T. Lee-Chiong, J. Pancer, and T. Swick, "Practice parameters for the use of actigraphy in the assessment of sleep and sleep disorders: An update for 2007,' Sleep, vol. 30, no. 4, pp. 519-529, 2007.

[16] J. R. Shambroom, S. E. Fábregas, and J. Johnstone, "Validation of an automated wireless system to monitor sleep in healthy adults," Journal of Sleep Research, vol. 21, no. 2, pp. 221-230, 2012.

[17] R. Parikh, M. N. Touvelle, H. Wang, and S. N. Zallek, "Sleep telemedicine: patient satisfaction and treatment adherence," Telemedicine and E-Health, vol. 17, no. 8, pp. 609-614, 2011.

[18] R. Spaulding, D. Stevens, and S. E. Velasquez, "Experience with telehealth for sleep monitoring and sleep laboratory management," Journal of Telemedicine and Telecare, vol. 17, no. 7, pp. 346-349, 2011.

[19] J. Paalasmaa and M. Ranta, "Detecting heartbeats in the ballistocardiogram with clustering," in Proceedings of the ICML/UAI/COLT 2008 Workshop on Machine Learning for Health-Care Applications, Helsinki, Finland, 2008.

[20] J. Paalasmaa, "A respiratory latent variable model for mechanically measured heartbeats," Physiological Measurement, vol. 31, no. 10, pp. 1331-1344, 2010.

[21] O. T. Inan, M. Etemadi, R. M. Wiard, G. T. A. Kovacs, and L. Giovangrandi, "Novel methods for estimating the ballistocardiogram signal using a simultaneously acquired electrocardiogram," in 31st Annual International Conference of the IEEE Engineering in Medicine and Biology Society, 2009, pp. 5334-5347.

[22] M. Sakakibara, T. Kanematsu, F. Yasuma, and J. Hayano, "Impact of real-world stress on cardiorespiratory resting function during sleep in daily life," Psychophysiology, vol. 45, no. 4, pp. 667-670, 2008.

[23] P. M. Gollwitzer, "Goal achievement: The role of intentions," European Review of Social Psychology, vol. 4, no. 1, pp. 141-185, 1993.

[24] O. Polo, L. Brissaud, B. Sales, A. Besset, and M. Billiard, "The validity of the static charge sensitive bed in detecting obstructive sleep apnoeas," European Respiratory Journal, vol. 1, no. 4, pp. 330-336, 1988. 